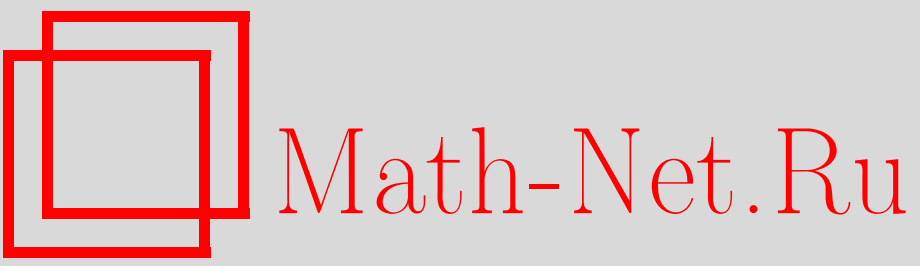

В. А. Зорич, В. М. Кесельман, Канонический вид изопериметрического неравенства на многообразиях конформно-гиперболического типа, УМН, 1999, том 54, выпуск 3, 165-166

DOI: https://doi.org/10.4213/rm175

Использование Общероссийского математического портала Math-Net.Ru подразумевает, что вы прочитали и согласны с пользовательским соглашением

http: //www . mathnet.ru/rus/agreement

Параметры загрузки :

IP : 35.174 .16 .151

26 апреля 2023 г., 18:16:58 


\title{
КАНОНИЧЕСКИЙ ВИД ИЗОПЕРИМЕТРИЧЕСКОГО НЕРАВЕНСТВА НА МНОГООБРАЗИЯХ КОНФОРМНО-ГИПЕРБОЛИЧЕСКОГО ТИПА
}

\author{
В. А. ЗОРИч, В. М. КЕСЕЛЬМАН
}

1. Введение. Как известно, изопериметрическое неравенство - это соотношение вида

$$
P(V(D)) \leqslant S(\partial D)
$$

между объемом $V(D)$ области $D$ и площадью $S(\partial D)$ ее границы, где $P$ - функция, назьваемая изопериметрической функцией пространства. Конечно, интерес представляет только нетривиальная, а чаще всего, даже максимальная изопериметрическая функция. Например, для евклидова $n$-мерного пространства $R^{n}$ таковой, с точностью до множителя, является функция $P(x)=x^{(n-1) / n}$, а для пространства Лобачевского - линейная функция $P(x)=x$.

Подобно пространствам Евклида и Лобачевского, произвольные открытые римановы (и даже субримановы) многообразия можно конформно-инвариантно разделить на два класса в соответствии с тем, какова конформная емкость абсолюта (бесконечности) этого многоообразия. Если эта емкость нулевая (как у пространства Евклида), мноогобразие относится к конформно-параболическому типу; если эта емкость положительна (как в случае пространства Лобачевского), то многообразие относится к конформно-гиперболическому типу.

Конформная замена метрики (что на физическом языке означает калибровочное преобразование, связанное с произволом в выборе местных масштабов) не меняет конформного типа многообразия, но меняет метрические соотношения на нем.

Нас интересует канонический вид изопериметрического соотношения в конформном классе метрики многообразия, точнее, вопрос о том, к какому каноническому виду приводится изпериметрическое неравенство конформными заменами метрики многообразия.

Результаты, полученные нами в [1], позволили высказать следующую рабочую гипотезу: на любом многообразии конформно-гиперболического типа максимальная изопериметрическая функция приводится к тому же линейному виду $P(x)=x$, что и в пространстве Лобачевского; а на любом многообразии конформно-параболического типа - к тому же виду $P(x)=x^{(n-1) / n}$, что и в пространстве Евклида.

Здесь мы рассмотрим первую часть этой гипотезы и дадим положительный ответ на вопрос, поставленньй в работе [2].

2. Конформный тип многообразия. Пусть $G$ - область на открытом римановом многообразии $(M, g)$, а $C$ - отличный от точки континуум (связный компакт) в $G$. Пару $(C, G)$ часто называют конденсатором, имея в виду, что $G \backslash C$ является полем конденсатора, а составляющие его границу $\partial(G \backslash C)$ множества $\partial G$ и $\partial C$ являются обкладками этого конденсатора.

Конформной емкостью конденсатора $(C, G)$ называется величина

$$
\operatorname{cap}(C, G)=\inf \int_{G}|\nabla f|^{n} d v
$$

где нижняя грань берется по всем гладким финитным в $G$ функциям $f$ таким, что $f \equiv 1$ на $C$ и $0 \leqslant f \leqslant 1$ в $G$.

Произвольную риманову метрику $\tilde{g}$ на $M$ называют конформно-эквивалентной или конформной метрике $g$, если $\tilde{g}=\lambda^{2} g$ на $M$, где $\lambda$ - некоторая положительная регулярная функция на $M$. Легко видеть (и это хорошо известно), что величина сар $(C, G)$ инвариантна относительно конформных замен исходной метрики многообразия.

С помощью емкости можно конформно-инвариантно охарактеризовать массивность абсолюта - идеальной границы многообразия. С этой целюю рассмотрим конденсаторы $(C, G)$, у которых внутренняя обкладка $\partial C$ фиксирована, а внешняя обкладка $\partial G$ уводится в "бесконечность", т.е. область $G$ расширяется до всего многообразия $M$. Предел емкостей таких конденсаторов совпадает со значением емкости сар $(C, M)$. При этом для данного многообразия $M$ величина

Работа выполнена при поддержке Российского фонда фундаментальных исследований (грант № 99-01-01179). 
сар $(C, M)$ либо положительна, либо равна нулю независимо от выбора неворожденного континуума $C$.

Таким образом, указанное свойство обращения или необращения в нуль емкости сар $(C, M)$ определяется только геометрией многообразия $M$ на "бесконечности" и в этом смысле часто говорят о нулевой или положительной конформной емкости абсолюта многообразия. Например, для евклидова пространства $\operatorname{cap}\left(C, R^{n}\right)=0$, а для пространства Лобачевского сар $\left(C, H^{n}\right)>0$.

Многообразие $M$ будем называть многообразием конформно-параболического типа, если конформная емкость его абсолюта равна нулю, т.е. $\operatorname{cap}(C, M)=0$ для любого невырожденного континуума $C \subset M$; если же эта емкость положительна, то будем называть $M$ многообразием конформно-гиперболического типа.

Такое разделение многообразий по указанным конформным типам, очевидно, инвариантно относительно конформных замен римановой метрики многообразия.

3. Формулировка основных результатов. Далее рассматривается произвольное $\boldsymbol{n}$-мерное некомпактное и без края риманово многообразие $M$ с некоторой исходной метрикой $g$. При переходе от метрики $g$ к конформной ей метрике $\tilde{g}$ одноименные геометрические объекты будут наделяться тильдой.

Теорема. Риманово многообразие $(M, g)$ имеет конформно-гиперболический тип тогда и только тогда, когда существует конформно-эквивалентная метрике $g$ риманова метрика $\tilde{g}$, в которой выполняется изопериметрическое неравенство:

$$
\widetilde{V}(D) \leqslant \widetilde{S}(\partial D)
$$

для всех регулярньх областей $D \subset M$, обгем $\widetilde{V}(D)$ которых больше фиксированного (произвольно малого) положительного числа.

Здесь под регулярной областью понимается область с кусочно-гладкой границей, имеющая конечный $n$-мерньй объем $\widetilde{V}(D)$ и конечную $(n-1)$-меру Хаусдорфа $\widetilde{S}(\partial D)$ границы области.

Таким образом, в конформном классе метрики риманова многообразия конформно-гиперболического типа изопериметрическая функция приводится к тому же линейному виду $P(x)=x$, что и в пространстве Лобачевского.

ДополнениЕ 1. Указанное в теореме неравенство $(*)$ является асимптотически точным в том смысле, что отношение величин $\widetilde{V}(D)$ и $\widetilde{S}(\partial D)$ может бить сколь угодно близким кединице для регулярных областей сколь угодно больиого $\tilde{g}$-обвема.

ДополнениЕ 2. Указанную в теореме метрику $\tilde{g}$ можно выбрать так, что обвем геодезического шара радиуса $r$ в этой метрике имеет порядок величины $e^{r}$ nри $r \rightarrow$ $+\infty$.

Напомним [3], что изопериметрической размерностью многообразия бесконечного объема называется точная верхняя грань всех чисел $m>0$, для каждого из которых функция $P(x)=$ $x^{(m-1) / m}$ (при всех $x>\varepsilon>0$ ) является изопериметрической функцией многообразия. Точную верхнюю грань изопериметрических размерностей многообразия по всем метрикам, конформным исходной метрике многообразия, будем называть (см. [4]) конформной изопериметрической размерностью многообразия. Используя сформулированную выше теорему, можно получить

СледствиЕ. Риманово многообразие $М$ имеет конформно-гиперболический тип тогда и только тогда, когда его конформная изопериметрическая размерность равна $+\infty$.

\section{СПИСОК ЛИТЕРАТУРЫ}

[1] Зорич В. А., Кесельман В. М. // Функц. анализ и его прил. 1996. V. 30. № 2. Р. 40-55. [2] Grimaldi R., Pansu P. // J. Math. Pures Appl. 1992. V. 71. № 1. P. 1-19. [3] Gromov M. // Textes Mathematiques, 1. Paris: Cedic/Fernand Nathan, 1981. [4] Зорич В. А., Кесельман В. М. // Матем. заметки. 1998. Т. 63. № 3. С. 379-385.

Московский государственный университет им. М. В. Ломоносова; Кемеровский государственный университет E-mail: vzor@glasnet.ru, kvm@kvm.kemerovo.su
Принято редколлегией 02.06.1999 